\title{
Malagasy Control and Its Theoretical Implications*
}

\author{
MARIA POLINSKY and ERIC POTSDAM \\ University of California, San Diego and University of Florida
}

\section{Introduction}

Few syntactic phenomena have attracted as much attention as Control: a structure in which the overt subject of a dominating clause (the controller) determines the referential properties of an unpronounced subject of its complement clause (the controllee). More than thirty years of research, starting with Rosenbaum (1967), Postal (1970), and Bresnan (1972), have produced several interesting theories of Control and Raising (for a good summary of approaches, see Davies and Dubinsky 2004). At the same time, most studies of Control have built heavily on the facts of English and a small number of other well-studied languages. The goal of this paper is to investigate Control in Malagasy, an Austronesian language spoken in Madagascar that is significantly different from English. We will present and analyze three Subject Control constructions in Malagasy which may provide an argument in favor of a syntactic analysis of Control as movement (Hornstein 1999, 2003). The paper is structured as follows. Section 1 introduces basic facts of Malagasy grammar. Section 2 briefly surveys the contrasting syntactic approaches of Control that we consider. Sections 3 through 6 describe and analyze three different patterns of Control in Malagasy, using two of the patterns to argue for the movement analysis. Section 7 summarizes the results of this work.

\section{Malagasy}

Malagasy has basic VOS word order and an articulated voice system. For our purposes, it is sufficient to recognize three voices shown in (1a-c): active or agent-topic, passive or theme-topic, and circumstantial (passivized applicative) which serves to promote an element other than agent or theme. In (1c), it is a beneficiary.

\footnotetext{
* This research was supported by NSF grants BCS-0131946 and BCS-0131993 and by a University of California Academic Senate grant. We are very grateful to our language consultants, Tina Boltz, Noro Brady, Solange Green, Cecile Manorohanta, Landy Rahelison, Charlotte AbelRatovo, Hasina Randriamihamina, and Annie Rasoanaivo. We have benefited from discussions with Gabriella Hermon, Ed Keenan, Matt Pearson, Roger-Bruno Rabenilaina, Lisa Travis and the audience at BLS 30. All errors are our sole responsibility.
} 

(1) a. n-i-vidy ny akoho (hoan-dRasoa) Rabe PAST-ACT(IVE)-buy the chicken for-Rasoa Rabe
'Rabe bought a chicken (for Rasoa).'
b. no-vidi-n-dRabe
(hoan-dRasoa) ny akoho PAST-buy-PASS(IVE)-Rabe for-Rasoa the chicken 'The chicken was bought (for Rasoa) by Rabe.'
c. n-i-vidi-anan-dRabe ny akoho Rasoa
PAST-ACT-buy-CIRC-Rabe the chicken Rasoa
'Rasoa was bought a chicken by Rabe.'
ACTIVE
PASSIVE

There are several proposals concerning the structure of such clauses and we will adopt structures from Guilfoyle, Hung, and Travis (1992), shown in (2). The clause-final subject occupies the specifier of IP and non-subject agents occur post-verbally in the specifier of VP.

(2)

a.

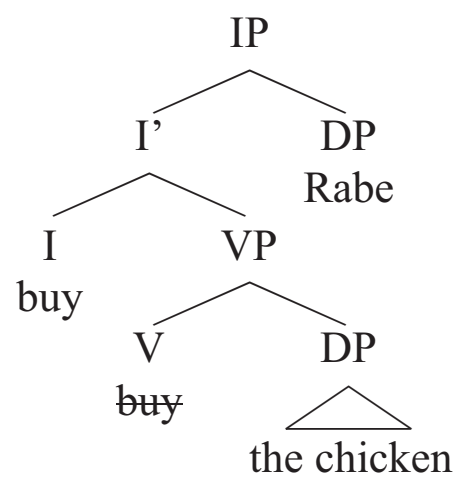

b.

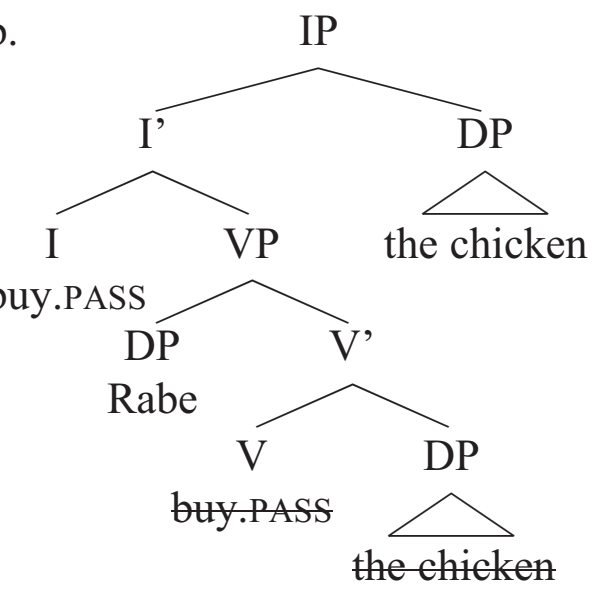

The voice system has an important syntactic function. As pointed out by many researchers, only the subject can be extracted for purposes of relativization, whquestioning, or topicalization (Keenan 1972, 1976, 1995, MacLaughlin 1995, Paul 1999, 2002, Pearson 2001, Sabel 2002, and many others). This is illustrated briefly in (3), which shows that only subject wh-questions are grammatical.

(3) a. iza no nividy ny akoho iza?

who FOCUS buy.ACT the chicken

'Who bought the chicken?'

b. *inona no nividy inona i Rabe?

what FOCUS buy.ACT Rabe

('What did Rabe buy?')

\section{Syntactic Approaches to Control}

With section 1 as background, we turn to the syntax of Control and Control in Malagasy. While we cannot do justice to the richness of various approaches to 
Control here, we will introduce two competing analyses: a base-generation approach and the more recent Minimalist-oriented movement account.

Within the Principles and Parameters theory (Chomsky and Lasnik 1993), the controllee in a Control structure is the null element PRO and it is co-indexed with the controller, as shown in (4).

(4) The farmer ${ }_{i}$ tried $\mathrm{PRO}_{i}$ to sell the cow

The PRO-based account rests on the theoretical assumptions in (5), among others. The Theta Criterion in (5a) forces the existence of PRO. (5b) restricts PRO's distribution to the subject of non-finite clauses and (5c) helps to determine PRO's interpretation.

(5) a. an NP chain may receive at most one $\theta$-role (part of the Theta Criterion)

b. PRO is assigned Null Case

c. PRO must be bound for a referential interpretation

Each of these assumptions has been questioned in the literature. Hornstein's (1999, 2003) Minimalist analysis of Control replaces them with the following:

(6) a. an NP chain may receive multiple $\theta$-roles

b. PRO does not exist

c. the controllee is a trace of NP-movement

Adopting these arguably Minimalist assumptions leads to a unification of Raising and Control. Both are derived via A-movement and they differ minimally in whether or not the higher predicate assigns a $\theta$-role to the raised NP. On Hornstein's analysis, a Control structure has the following derivation:

(7) The farmer tried the farmer to sell the cow

Hornstein 1999 discusses a number of conceptual advantages to the movement analysis. In what follows, we introduce three Malagasy control structures in an effort to provide new empirical data which might contribute to this theoretical debate. ${ }^{1}$

\section{Active Control}

ACTIVE CONTROL, in (8), is the Malagasy construction that most closely resembles English Control. The control predicate appears in the active voice, while the voice of the embedded predicate is not restricted.

\footnotetext{
${ }^{1}$ Previous research on Malagasy Control constructions include Keenan (1976, 1995), Law (1995), Paul and Ranaivoson (1998), Pearson (2001), and Polinsky and Potsdam (2002a, 2003).
} 


\section{Maria Polinsky and Eric Potsdam}

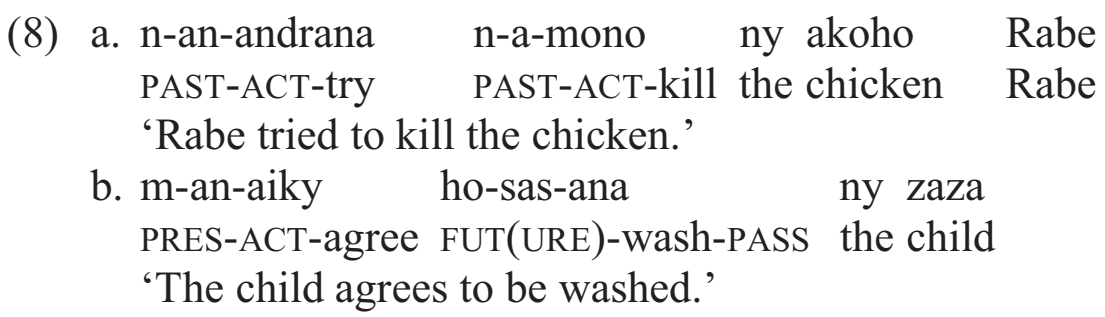

This construction is accepted by all speakers and has properties typical of a Subject Control structure. The matrix verb imposes selectional restrictions on its subject and there is an obligatory control interpretation with the embedded subject position, which must remain unexpressed. In brief, the Active Control construction is unsurprising in resembling English and other well-known languages and, as a consequence, it does not shed light on the theoretical debate between basegeneration and movement analyses of the phenomenon.

\section{Backward Control}

BACKWARD CONTROL is the apparently similar construction illustrated in (9). It seems limited to three verbs (mahavita 'accomplish', mitsahatra 'stop', and manomboka 'begin') and is subject to unpredictable idiolectal variation.
a. n-a-havita
namono
ny akoho Rabe
PAST-ACT-accomplish kill.ACT the chicken Rabe
'Rabe finished killing the chicken.'
b. m-an-omboka mitondra ny fiara Rabe
PRES-ACT-begin drive.ACT the car Rabe
'Rabe is beginning to drive the car'

As with Active Control, the control predicate is in the active voice and it imposes selectional restrictions on the overt subject. For example, these verbs do not allow non-volitional subjects, (10), and they form an imperative, (11) (Perlmutter 1970).

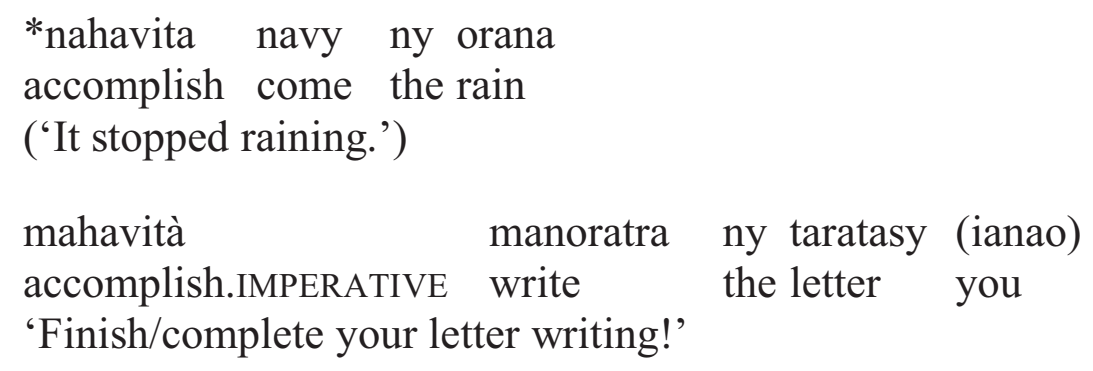

$\begin{array}{llll}\text { mahavità } & \text { manoratra } & \text { ny taratasy (ianao) } \\ \text { accomplish.IMPERATIVE } & \text { write } & \text { the letter you } \\ \text { 'Finish/complete your letter writing!' } & & \end{array}$

There is also an obligatory control interpretation between the two subject arguments and (9) cannot mean 'Rabe finished having someone kill the chicken'. Similarly, the two subject positions cannot be simultaneously expressed, (12). 

(12) a. *n-a-havita namono ny akoho izy/azy Rabe PAST-ACT-accomplish kill.ACT the chicken 3SG.NOM/3SG.ACC Rabe ('Rabe finished killing the chicken.')

In contrast to Active Control, however, the construction has the unusual constituency shown in (13) in which the overt subject is in the embedded clause. It is not the subject of the matrix control predicate.
n-a-havita
[namono ny akoho
Rabe]
PAST-ACT-accomplish kill.ACT the chicken Rabe
'Rabe finished killing the chicken.'

Evidence for this constituency comes from coordination (see Polinsky and Potsdam 2002a for more detailed argumentation). The matrix verb and embedded predicate cannot coordinate to the exclusion of the subject, (14a). Instead, the embedded subject must be repeated, (14b). This is expected given the constituency in (13). If the overt subject were outside the embedded clause, (14a) should be possible.

$\begin{array}{cllll}\text { (14) a. *nanomboka namaky ny taratasy sy menatra } & \text { ny mpianatra } \\ \text { began } & \text { read the letter and embarrassed } & \text { the student }\end{array}$ ('The student began to read the letter and was embarrassed.')

b. nanomboka namaky ny taratasy ny mpianatra ka menahatra izy began read the letter the student and embarrassed 3SG 'The student began to read the letter and he was embarrassed.'

Given that the overt subject is in the embedded clause but the matrix predicate imposes selectional restrictions, there must be a non-overt subject in the higher clause coindexed with the lower subject. We represent this controllee atheoretically as $\Delta$ in (15).

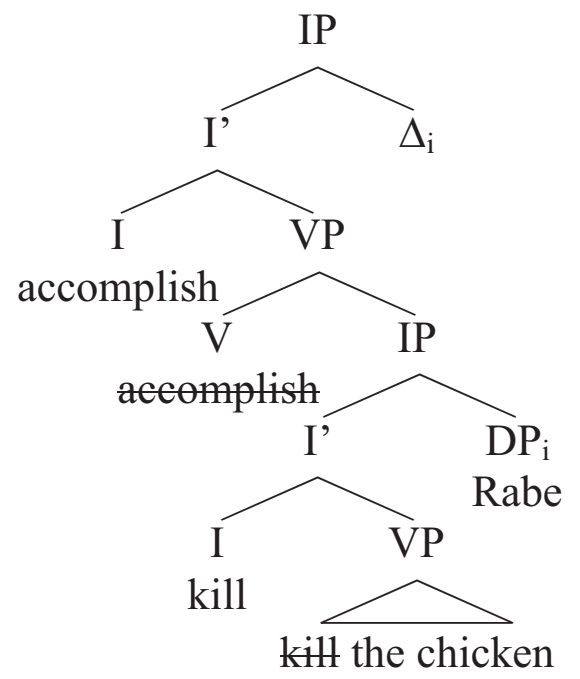


Additional evidence for this null controllee comes from quantifier float. Floated daholo 'all' is licensed under c-command in the same clause as its binder (Keenan 1976, 1995), (16a). (16b) shows that daholo 'all' may also appear in the matrix clause in Backward Control constructions. This is unexpected unless there is a null controllee in the matrix clause.

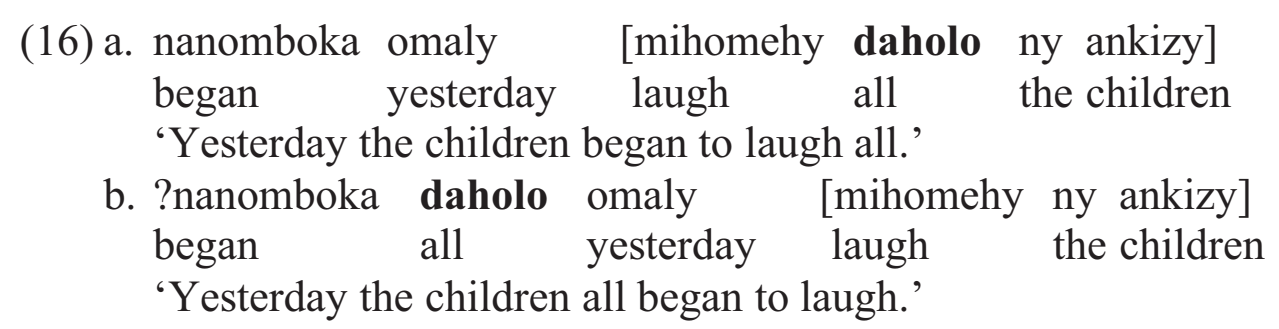

In summary, as we have argued in more detail elsewhere (Polinsky and Potsdam 2002a), this construction instantiates Backward Subject Control, a control construction in which the structural positions of the controller and controllee are reversed. It is also found in Tsez (Polinsky and Potsdam 2002b), Tsaxur (Kibrik 1999), and possibly Kabardian (Kumaxov and Vamling 1998) and Adyghe (Say 2004).

Turning now to the syntactic analysis of Backward Control, it clearly presents a problem for the base-generation analysis:

$$
\text { [accomplish [kill the chicken } \left.\left.\mathrm{Rabe}_{\mathrm{i}}\right] \quad \Delta_{\mathrm{i}}\right]
$$

If the matrix subject is PRO, it is not bound and the sentence should, instead, receive an arbitrary interpretation, contrary to fact. At the same time, with coindexing, the structure is a violation of Binding Theory Condition $\mathrm{C}$, since the $\mathrm{R}$-expression Rabe is not free. This should rule out the structure on a control interpretation.

There are similar problems if the matrix subject is the null pronominal pro. First, Malagasy is not a pro-drop language. Second, even if pro were exceptionally present in this particular configuration, the obligatory control interpretation is unexpected. Third, the presence of pro would again lead to condition $\mathrm{C}$ violation. Finally, it is surprising to find a null pronominal that never alternates with an overt NP, (12).

The conclusion that the controllee is not a base-generated empty category suggests that we consider Hornstein's movement analysis of control discussed in section 2. In Polinsky and Potsdam $(2002 \mathrm{a}, \mathrm{b})$ we propose in more detail that Backward Control differs from Forward Control only in that the raising of the controller takes place in the covert syntax:

(18) derivation of Backward Control

a. [IP [VP accomplish [IP Rabe [VP kill chicken]]]] SS

b. [IP Rabe [VP accomplish [IP Rabe [VP kill chicken]]]] 
This approach correctly derives the relevant Malagasy construction and avoids the analytical problems that accompany the empty category analysis. If this approach can be maintained, the Backward Control construction offers support for a derivational view of Control. In the next section, we turn to a construction that seems to pose a challenge to this conclusion.

\section{Passive Control}

The third control construction that we discuss is PASSIVE CONTROL, as in (19). It involves a passive control predicate and a passive or circumstantial verb in the embedded clause. Both the controller and controllee are passive agents.

(19) a. n-andram-an-dRabe no-vono-ina ny akoho PAST-try-PASS-Rabe PAST-kill-PASS the chicken (lit.: the chicken was tried by Rabe to be killed) 'Rabe tried to kill the chicken.'

b. kasa-in-dRasoa ho-sas-ana ny zaza intend-PASS-Rasoa FUT-wash-PASS the child (lit.: the child is intended by Rasoa to be washed) 'Rasoa intends to wash the child.'

As before, the control predicate imposes selectional restrictions on its agent and the controllee cannot be expressed, (20).
$*_{n}$-andram-an-dRabe $e_{i}$
no-vono-i-ny
ny akoho PAST-try-PASS-Rabe PAST-kill-PASS-3SG the chicken ('Rabe tried to kill the chicken.')

The structure we posit for passive control is in (21) below. The matrix subject cyclically raises from the embedded clause, first undergoing passive and then subject-to-subject raising into the matrix subject position. The control relationship is established by movement from the lower spec, $\mathrm{V}$ to the higher.

This derivation however violates Relativized Minimality because it contains two overlapping A-chains. This challenges the analysis of Control as movement. At this juncture we can entertain three analytical possibilities: (i) the PRO-based analysis should be revived, (ii) the construction is Non-Obligatory Control and, as such, it is not analyzed as movement under Hornstein's (1999) theory, or (iii) there is a different analysis in terms of movement compatible with Relativized Minimality. The phenomenon of Backward Control forces us to reject (i). In the section to follow, we explore (ii) in more detail and ultimately reject it. We sketch a solution along the lines of (iii) based on work in progress. 
Maria Polinsky and Eric Potsdam

(21)

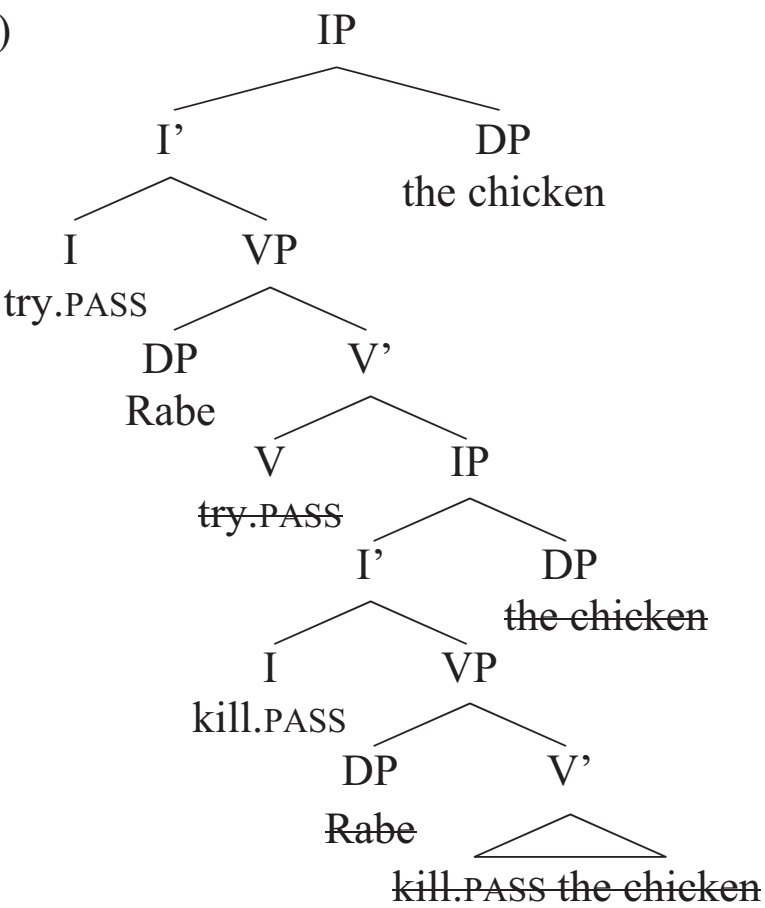

\section{Obligatory and Non-Obligatory Control}

Many researchers have recognized and investigated the difference between Obligatory Control (OC) and Non-obligatory Control (NOC) illustrated in (22) (see Jackendoff and Culicover 2003 for a discussion). The controller in OC must be very local while the choice of controller in NOC is more open.

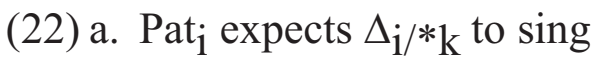
OBLIGATORY

b. Pat ${ }_{\mathrm{i}}$ thinks that $\Delta_{\mathrm{i}+\mathrm{k} / \mathrm{k}}$ to sing would be fun

NON-OBLIGATORY

Hornstein (1999) proposes that the two constructions have different syntactic structures. Only OC involves movement; NOC is a base-generated structure. If Malagasy passive control were NOC, it would not pose a problem for the movement analysis of control - it would be simply irrelevant to it.

OC and NOC differ in a number of documented ways (Hornstein 1999, 2003, Jackendoff and Culicover 2003, and references therein):

properties of OC versus NOC

a. no controller $\left(\mathrm{PRO}_{\text {arb }}\right.$ reading)

b. permits strict reading under ellipsis

c. paraphrasable with a pronoun

d. allows a non-local antecedent

e. allows a non-c-commanding antecedent

$\begin{array}{cc}\text { OC } & \text { NOC } \\ x & \sqrt{ } \\ x & \sqrt{ } \\ x & \sqrt{ } \\ x & \sqrt{ } \\ x & \sqrt{ }\end{array}$

We will now apply these diagnostics to Malagasy Passive Control. For comparison, we also present data on Active Control, an uncontroversial OC construction. 
What the data below show is that all properties identify Passive Control as OC, no different from Active Control. ${ }^{2}$

(24) no controller, $\mathrm{PRO}_{\text {arb }}$ reading
a. nanaiky hividy ity trano ity Rabe agree.ACT buy.ACT this house this Rabe
b. neken' i Rabe hovidina ity trano ity agree.PASS' Rabe buy.PASS this house this
*'Rabe agreed for someone to buy this house.'
'Rabe agreed to buy this house.'

(25) sloppy vs. strict reading under ellipsis:

a. nanaiky hividy ny trano ny mpitsara.Ilay mpampiasa koa. ACTIVE
agree buy the house the judge this employer too

b. neken'ny mpitsara hovidina ny trano. Ilay mpampiasa koa PASSIVE agree.PASS'the judge buy.PASS the house this employer too

'The judge agreed to buy the house. The employer (agreed to buy it) too.'

SLOPPY

*'The judge agreed to buy the house. The employer (agreed for the judge to buy it) too.'

*STRICT

(26) paraphrasable with a pronoun
a. \%nanaiky azy hividy ilay trano ny mpitsara agree.ACT 3SG buy.ACT this house the judge
b. neken' ny mpitsara hovidi-ny ilay trano agree.PASS' the judge buy.PASS-3SG this house
*'The judge agreed to buy this house.'
'The judge agreed for him (someone else) to buy this house.'

(27) non-local antecedent
a. mihevitra Rabe fa nanaiky hividy ny fiara Rasoa ACTIVE think Rabe that agree.ACT buy.ACT the car Rasoa
b. mihevitra Rabe fa neken-dRasoa hovidina ny fiara PASSIVE think Rabe that agree.PASS-Rasoa buy.PASS the car 'Rabe thinks that Rasoa agreed to buy the car.'

*'Rabe thinks that Rasoa agreed for him (Rabe) to buy the car.'

\footnotetext{
${ }^{2}$ In earlier work (Polinsky and Potsdam 2003) and in the presentation of this paper, we claimed that Passive Control was NOC. That claim was based on data from a smaller number of speakers and showed variability among speakers and graded judgments. The current conclusion is based on more extensive fieldwork in Madagascar with a larger set of consultants, who seem to be more in agreement with each other and rarely show graded judgments. At the same time, there does seem to be variation in judgments with different predicates which we have not pursued. For illustration, we have used the predicate manaiky 'agree', which shows clear OC behavior.
} 
(28)

non-c-commanding antecedent:

a. nanaiky hividy ny kadoa agree.ACT buy.ACT the gift the children' Rasoa

b. neken' ny zanak' i Rasoa hovidina ny kadoa PASSIVE agree.PASS' the children' Rasoa buy.PASS the gift

'Rasoa's children agreed to buy a gift.'

*'Rasoa's children agreed for her (Rasoa) to buy a gift.'

Given that Passive Control is OC, the analysis according to which passive control is base-generated as NOC is untenable. This leaves us with the need to re-evaluate the movement analysis.

In ongoing work, we are pursuing the idea that the overlapping chains of movement in (21) are allowed because they instantiate different kinds of chains. The movement of the controller from spec, $\mathrm{V}$ to $\mathrm{spec}, \mathrm{V}$ is $\mathrm{A}$-movement but the movement of the theme from spec,I to spec, I is in fact $A^{\prime}$-movement. This proposal relies on a particular view of Malagasy clause structure stated in (29).

(29) a. the post-verbal NP is the subject

b. the clause-final NP is an obligatory topic in an A'-position

That the clause-final NP must be specific (i.e. a topic) in Malagasy is well-known (see for example Keenan 1976, Pearson 1996, 2001, and Paul 2000). This view of Malagasy grammar is most recently and forcefully defended in Pearson (to appear) and the existence of passive control may further support this position.

\section{Conclusions}

In this paper, we have considered three control constructions in Malagasy: Active Control, Backward Control, and Passive Control. Examination of these constructions shows that the range of variation in Malagasy Control is richer than would be predicted on the basis of English and similar languages.

While expanding the empirical database of control structures available crosslinguistically, Malagasy Control constructions also validate fundamental properties of Control structures proposed on the basis of more familiar languages. In particular, the seemingly unusual Passive Control construction shows all the standard properties that identify Obligatory Control.

The Active Forward construction does not differ from well-known Subject Control in English and as such does not inform the ongoing theoretical debate concerning the optimal model for Control structures. The other two constructions discussed in this paper offer new empirical evidence for the derivational analysis of Control. This evidence crucially relies on internal facts of Malagasy grammar. 


\section{References}

Bresnan, Joan. 1972. Theory of complementation in English syntax. PhD dissertation, MIT.

Chomsky, Noam, and Howard Lasnik. 1993. The theory of Principles and Parameters. In J. Jacobs, A. von Stechow, W. Sternefeld, and T. Vennemann (eds.) Syntax: An International Handbook of Contemporary Research. Berlin: Mouton.

Davies, William, and Stanley Dubinsky. 2004. A Grammar of Raising and Control. Oxford: Blackwell.

Guilfoyle, Eithne, Henrietta Hung, and Lisa Travis. 1992. Spec of IP and Spec of VP: Two subjects in Austronesian languages. Natural Language and Linguistic Theory 10: 375-414.

Hornstein, Norbert. 1999. Movement and control. Linguistic Inquiry 30: 69-96.

Hornstein, Norbert. 2003. On Control. In R. Hendrick (ed.) Minimalist Syntax, 681. Oxford: Blackwell.

Jackendoff, Ray, and Peter Culicover. 2003. The semantic basis of control in English. Language 79: 517-556.

Keenan, Edward L. 1972. Relative clause formation in Malagasy. In P. M. Peranteau, J. N. Levi, and G. C. Phares (eds.) The Chicago Which Hunt: Papers from the Relative Clause Festival, 169-189. Chicago: Chicago Linguistic Society.

Keenan, Edward L. 1976. Remarkable subjects in Malagasy. In C. N. Li (ed.). Subject and Topic, 247-301. New York: Academic Press.

Keenan, Edward L. 1995. Predicate-argument structure in Malagasy. In C. S. Burgess, K. Dziwirek, and D. Gerdts (eds.) Grammatical Relations: Theoretical Approaches to Empirical Questions, 171-216. Stanford: CSLI.

Kibrik, Alexander E. (ed.). 1999. Elementy caxurskogo jazyka v tipologičeskom osveščenii [Aspects of Tsaxur Grammar From a Cross-Linguistic Perspective]. Moscow: Nasledie.

Kumaxov, Muxadin, and Karina Vamling. 1998. Complementation in Kabardian. Lund: Lund University Publications.

Law, Paul. 1995. On grammatical relations in Malagasy control structures. In C. S. Burgess, K. Dziwirek, and D. Gerdts (eds.) Grammatical Relations: Theoretical Approaches to Empirical Questions, 271-290. Stanford: CSLI.

MacLaughlin, Dawn. 1995. Wh-movement in Malagasy: An extraction asymmetry. In A. Akinlabi (ed.) Theoretical Approaches to African Linguistics, 117128. Trenton, NJ: Africa World Press.

Paul, Ileana. 1999. Malagasy clause structure. PhD dissertation, McGill University.

Paul, Ileana. 2000. Malagasy existentials: A syntactic account of specificity. In Ileana Paul, Viviane Phillips, and Lisa Travis (eds.) Formal Issues in Austronesian Linguistics, 65-83. Dordrecht: Kluwer. 
Paul, Ileana. 2002. On extraction asymmetries. In MIT Working Papers in Linguistics 44: The Proceedings of AFLA XIII, 211-224.

Paul, Ileana and Jeannot F. Ranaivoson. 1998. Complex verbal constructions in Malagasy. In I. Paul (ed.) The Structure of Malagasy II. UCLA Occasional Papers in Linguistics 20, 111-125.

Pearson, Matthew. 1996. Domain phrases and topic arguments in Malagasy existentials. In Matthew Pearson and Ileana Paul (eds.) UCLA Occasional Papers in Linguistics 17: The Structure of Malagasy, 113-141. Los Angeles: UCLA Department of Linguistics.

Pearson, Matthew. 2001. The clause structure of Malagasy: A Minimalist approach. PhD dissertation, UCLA.

Pearson, Matthew. To appear. The Malagasy subject/topic as an A'-element. Natural Language and Linguistic Theory.

Perlmutter, David. 1970. The two verbs begin. In E. Jacobs and P. Rosenbaum (eds.) Readings in English Transformational Grammar, 107-119. Waltham, MA: Blaisdell.

Polinsky, Maria, and Eric Potsdam. 2002a. Backward control: Evidence from Malagasy. In MIT Working Papers in Linguistics 44: The Proceedings of AFLA XIII, 257-272.

Polinsky, Maria, and Eric Potsdam. 2002b. Backward control. Linguistic Inquiry 33: 245-282.

Polinsky, Maria, and Eric Potsdam. 2003. Control in Malagasy. Cornell Working Papers in Linguistics 19: 173-187.

Postal, Paul M. 1970. On Raising. Cambridge, MA: MIT Press.

Rosenbaum, Peter. 1967. The Grammar of English Predicate Complement Constructions. Cambridge, MA: MIT Press.

Sabel, Joachim. 2002. Wh-questions and extraction asymmetries in Malagasy. In MIT Working Papers in Linguistics 44: The Proceedings of AFLA XIII, 309324.

Say, Sergey. 2004. On so-called Backward Control in Adyghe. Paper presented at the Conference on Control, ZAS, Berlin, May 2004.

Maria Polinsky

Linguistics Department

University of California, San Diego

La Jolla, CA 92093-0108

polinsky@ling.ucsd.edu
Eric Potsdam

Linguistics Program

University of Florida

Gainesville, FL 32611-5454

potsdam@ufl.edu 